\title{
The impact of sample size and marker selection on the study of haplotype structures
}

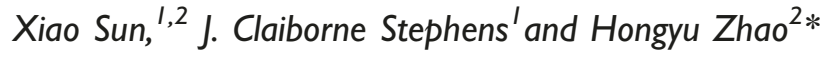 \\ 'Genaissance Pharmaceuticals, 5 Science Park, New Haven, CT 06511, USA \\ ${ }^{2}$ Yale University School of Medicine, 60 College Street, New Haven, CT 06520, USA \\ *Correspondence to: Tel: +1 203785 6271; Fax: +1 203785 6912, E-mail: hongyu.zhao@yale.edu
}

Date received (in revised form): 22nd December 2003

\begin{abstract}
Several studies of haplotype structures in the human genome in various populations have found that the human chromosomes are structured such that each chromosome can be divided into many blocks, within which there is limited haplotype diversity. In addition, only a few genetic markers in a putative block are needed to capture most of the diversity within a block. There has been no systematic empirical study of the effects of sample size and marker set on the identified block structures and representative marker sets, however. The purpose of this study was to conduct a detailed empirical study to examine such impacts. Towards this goal, we have analysed three representative autosomal regions from a large genome-wide study of haplotypes with samples consisting of African-Americans and samples consisting of Japanese and Chinese individuals. For both populations, we have found that the sample size and marker set have significant impact on the number of blocks and the total number of representative markers identified. The marker set in particular has very strong impacts, and our results indicate that the marker density in the original datasets may not be adequate to allow a meaningful characterisation of haplotype structures. In general, we conclude that we need a relatively large sample size and a very dense marker panel in the study of haplotype structures in human populations.
\end{abstract}

Keywords: single nucleotide polymorphism (SNP), haplotype, sample size, marker selection, haplotype block, tag SNPs

\section{Introduction}

Human DNA sequence variation accounts for a large fraction of the observed phenotypic differences between individuals, including susceptibility to disease. Sites in the DNA sequence where individuals differ at a single DNA base are called single nucleotide polymorphisms (SNPs). SNPs represent by far the most common source of genetic variation, and it is estimated that the human genome may contain over 10 million SNPs, about one in every 300 bases. $^{1-3}$

A haplotype is the specific combination of marker alleles within a region of a chromosome. Tightly linked SNPs are not independent on a given chromosome, but tend to be associated with each other across small regions. This tendency is called linkage disequilibrium. Empirical data suggest that relatively few of the theoretically possible haplotypes are observed at significant frequencies for a set of SNPs within a very short physical distance. ${ }^{4}$

Genome-wide disease association studies using SNPs and haplotypes may be the most promising approach to identifying genetic variants underlying complex diseases, and recent technological advances have made high-throughput sequencing and genotyping possible. With the aim of speeding the discovery of genes related to common illnesses, as well as preventing adverse drug reactions, the National Institutes of Health launched the international HapMap Project to organise what is known about genetic variation within the human genome. One objective of this project was to understand haplotype structures throughout the human genome.

Recent studies ${ }^{5-8}$ have shown that haplotypes may be divided into discrete blocks, within which there is limited haplotype diversity. For example, Gabriel and colleagues systematically examined 51 autosomal regions in four populations and found that the minimal span of the blocks averaged $9 \mathrm{~kb}$ in Yoruban and African-American samples, with a range of $<1 \mathrm{~kb}$ up to $94 \mathrm{~kb}$, whereas the average in European and Asian samples was $18 \mathrm{~kb}$, with a range of $<1 \mathrm{~kb}$ to $73 \mathrm{~kb}$. $^{8}$ Furthermore, in a study of the class II region of the major histocompatibility complex, researchers found that the haplotype blocks were flanked by precisely localised recombination hotspots, leading to the hypothesis that 'punctuate recombination' could be the molecular mechanism underlying block structure. ${ }^{9}$ 
One attractive feature of statistical association methods based on haplotype blocks is the idea that, although blocks may contain a large number of SNPs, only a few SNPs are needed to uniquely identify the haplotypes in a block. This much smaller subset of SNPs, which are termed 'haplotype tagging SNPs' (htSNPs), can be used to explain a large proportion of diversity. Tag SNPs make it unnecessary to genotype all the SNPs in a given region and therefore represent an economic approach to genome-wide association studies. Zhang et al. ${ }^{10}$ studied the power of different association tests in a variety of disease models by using Tag SNPs and concluded that the genotyping efforts can be significantly reduced without much loss of power.

Despite these findings of block-like structures in the human genome, there is no universally accepted definition of haplotype blocks. In fact, each study has its own definition. Different definitions of haplotype blocks include: (1) a continuous set of markers in which the average pairwise D' is greater than some predetermined threshold; ${ }^{11}$ (2) a region where a small number of common haplotypes account for the majority of the chromosomes; ${ }^{6,12}$ (3) regions with both limited haplotype diversity and strong linkage disequilibrium but allowing several markers to be skipped; ${ }^{7}$ and (4) regions with absolutely no evidence for historical recombination between any pair of SNPs. ${ }^{13}$ Therefore, block definition remains subjective and arbitrary, and it is not yet clear how to compare haplotype blocks between studies. Furthermore, each method varies in terms of the SNP minor allele frequency threshold used. The most appropriate definition may depend on how the inferred blocks are used, such as whether the identified blocks will be used to infer recombination hot spots or to identify regions that are associated with disease. Moreover, recent studies suggest that there may be non-trivial departures from block structures. ${ }^{14}$

Despite extensive empirical studies on haplotype blocks, one issue that has not been well addressed is the impact of sample size on the assessment of haplotype block structure. In some previous studies, blocks were identified based on a small set of chromosomes and may not provide a comprehensive representation of the whole population. For example, the chromosome 21 study only examined 20 independent chromosomes from diverse populations. ${ }^{6}$ The largest dataset reported to date contains samples from 275 individuals, leading to 400 independent chromosomes. ${ }^{8}$ It is not known, however, how many individuals are sufficient to get reliable characterisation of haplotype block structures.

In addition, the effect of SNP marker selection on the inferred haplotype block structures has not been well studied either. To date, the density of SNPs analysed has ranged from approximately one marker per kilobase ${ }^{6,9}$ to one marker per $15 \mathrm{~kb}^{7}$ Published results suggest that a denser marker panel tends to give rise to a larger number of shorter blocks, ${ }^{6}$ whereas a sparser marker panel generates fewer longer blocks. ${ }^{7,8}$ Furthermore, the block boundaries and Tag SNPs may substantially change, even if we keep the SNP density constant but select a different set of SNPs. In a recent study by Wall and Pritchard, ${ }^{15}$ they found using simulations that marker density is more important than sample size for inferring haplotype structures.

One of the objectives of the HapMap project is to understand population differences in their haplotype structures. It is important to compare haplotype blocks in different populations and to examine whether the same set of Tag SNPs can be used in different populations to capture haplotype diversities. Existing data have shown that the blocks in a Yoruban population from Nigeria are generally the same as, but shorter than, those in European and Asian populations. ${ }^{8}$ If different populations indeed share similar haplotype block structures, one broad map would be sufficient. If the populations are different enough, however, it might be necessary to construct population-specific haplotype maps.

These are very important questions requiring answers, and the data collected from the HapMap project may help us to gain a better understanding of these issues. In the current study, we focused on the impact of sample size and SNP marker selection on the haplotype block partitioning and Tag SNP selections in a sample consisting of African-Americans and a sample consisting of Japanese and Chinese people.

\section{Materials and methods}

\section{Datasets}

SNP genotype data of 51 autosomal regions that collectively span $\sim 0.4$ per cent of the human genome from AfricanAmerican samples (called population B in the original study) and from Japanese and Chinese samples (called population $\mathrm{C}$ in the original study) were downloaded from the following website: http://www.genome.wi.mit.edu/mpg/hapmap/ hapstruc.html. A detailed description of the data can be found in the paper by Gabriel et al., ${ }^{8}$ Population B contains 50 samples from unrelated African-Americans and population $\mathrm{C}$ includes 42 samples from unrelated individuals of Japanese and Chinese origin. This is the largest public dataset available to date.

In order to examine the impact of sample size and marker selection on haplotype block boundaries and Tag SNPs, three regions from the above database were chosen in our study. Region 52a spans $237.22 \mathrm{~kb}$ on chromosome 22 and contains 46 SNPs for population B and 45 SNPs for population C. Region 42a is $409.92 \mathrm{~kb}$ long and is located on chromosome 15, it includes 100 SNPs for population B and 99 SNPs for population C. Region 31a is the shortest of the three. It is on chromosome 9, is $181.98 \mathrm{~kb}$ long and has 23 SNPs for population B and $25 \mathrm{SNPs}$ for population C. The density of the markers in these three regions is one SNP per 4 to $8 \mathrm{~kb}$. We chose these three regions because they represent small, medium and large numbers of SNPs within a given region in this dataset. 


\section{Haplotype block partitioning and Tag SNP selections}

To obtain haplotype boundaries and Tag SNPs, we used 'HapBlock', a dynamic programming algorithm for haplotype block partitioning with minimum number of Tag SNPs developed by Zhang et al., ${ }^{12}$ The following parameters were used in our analysis: the input data type was genotype data; the method for block definition was the one used in Patil et al. ${ }^{6}$ the threshold to define the block was set at 0.8 ; the threshold to define the common haplotype was set at 0.099 ; the method to find the Tag SNPs was the haplotype block diversity introduced by Johnson et al.; ${ }^{16}$ and the threshold to find the Tag SNPs was set at 0.9.

\section{Impact of sample size}

To examine the impact of sample size on the identified haplotype structures, we randomly selected 10, 20, 30 and 40 individuals out of 50 African-Americans in population B and repeated the random selection 100 times. For each randomly selected sample, we took their SNP genotype data in regions 52a, 42a and 31a and ran the HapBlock program to identify the number of blocks, the block boundaries and the Tag SNPs for each block. The same procedures were applied to population $\mathrm{C}$, which included 42 unrelated Japanese and Chinese people. These results were used to assess the effect of sample size on haplotype block structures.

\section{Impact of marker selection}

Random marker selection. To study the impact of marker selection on the assessment of haplotype block structures, we carried out random selection on SNP markers for the three regions. Because region 52a contains $46 \mathrm{SNPs}$ for population B (African-American) and 45 SNPs for population C (Japanese and Chinese), we randomly selected 10, 20, 30 and 40 SNPs for each population and repeated random selection 100 times. For region 42a, which includes 100 SNPs for population B and 99 SNPs for population C, we randomly selected 20, 40, 60 and 80 SNPs for each population and repeated this 100 times. Similarly for region 31a, where there are 23 SNPs for population B and 25 SNPs for population C, we randomly selected 5, 10, 15 and 20 SNPs for each population and repeated this 100 times. For each marker set selected, we ran the HapBlock program to identify the total number of blocks, the block boundaries and the Tag SNPs for each block.

Sequential marker selection. Since an SNP could only be a boundary marker in the event that it was in the subset chosen, comparing block boundaries among totally different sets of SNP markers is difficult. In order to further investigate the underlying mechanism explaining why higher density markers usually give rise to more, smaller blocks than is the case for lower density markers, we applied a sequential marker selection method to $46 \mathrm{SNPs}$ on chromosome region 52a from the African-American population. First, we randomly selected ten SNPs out of the original 46 SNPs to identify block structures. Secondly, we randomly selected another ten SNPs out of the 36 remaining SNPs and combined them with the previously selected 10 SNPs to identify block structures. Then, we randomly selected another 10 SNPs out of the 26 remaining SNPs and combined them with the previously selected 20 SNPs to do the analysis. Lastly, we randomly selected 10 more SNPs out of the 16 remaining SNPs and combined them with the previously selected 30 SNPs to identify block structures. This simulation approach ensured that the lower density marker set is a subset of the higher density marker set. The whole selection process was repeated 100 times. Comparisons of the block boundary results were based on these results.

\section{Block boundary and Tag SNP comparisons}

In the comparison of block boundaries, we counted the frequency of each SNP that was used as the starting or ending position of the block boundaries in the results based on 100 randomly selected samples. Comparing Tag SNPs is more complicated than comparing block boundaries because the Tag SNPs are not unique for each block. In other words, there is usually more than one set of Tag SNPs (see Appendix A for a Tag SNP example) in a block. Therefore, to incorporate the multiplicities of the Tag SNPs, for the results from each randomly selected sample, we counted the frequency of each SNP that was selected as a Tag SNP across all Tag SNP sets and divided this frequency by the number of Tag SNP sets in each block and the total number of blocks in the region. Based on the 100 randomly selected samples, we then calculated the mean weighted frequency for each SNP.

\section{Results}

\section{Haplotype block partitioning based on the observed data}

Using the observed genotype data, region 52a was partitioned into nine blocks with a total of 19 Tag SNPs for the AfricanAmericans (population B) and six blocks with a total of ten Tag SNPs for the Japanese and Chinese (population C). Region 42a, however, was divided into 16 blocks with a total of 33 Tag SNPs for African-Americans and 14 blocks with a total of 22 SNPs for Japanese and Chinese. As with region 31a, both populations had three blocks and six Tag SNPs (see appendix for detailed block information using region 52a as an example).

Inspection of all 51 autosomal regions in the Gabriel et al. data set reveals that, in general, chromosomal regions were partitioned into more blocks and had more tag SNPs based on the African-American samples than those based on the Japanese and Chinese samples. In addition, for both populations, the total number of Tag SNPs increases as the number of blocks increases (data not shown). 


\section{Impact of sample size}

Table 1 summarises the results of the number of blocks when we randomly selected 10, 20, 30 and 40 individuals 100 times from each population. For example, in the upper left panel of Table 1, column 'ran10' corresponds to the results based on 100 simulated datasets consisting of ten individuals. The sum did not add up to 100 because the HapBlock program we used for block partitioning would tend to fail when

Table I. Frequency of the number of blocks in which the number of individuals is varied in simulations

Region 52a (Chromosome 22, $237.22 \mathrm{~kb}$ )

African-American

50 individuals, 46 SNPs, 9 blocks

\begin{tabular}{c|cccc|} 
\# blocks & ran10 & ran20 & $\operatorname{ran} 30$ & $\operatorname{ran} 40$ \\
10 & & 4 & 24 & 36 \\
9 & & 31 & 53 & 49 \\
8 & 6 & 49 & 23 & 15 \\
7 & 21 & 14 & & \\
6 & 55 & 2 & & \\
5 & 17 & & & \\
4 & & & & \\
3 & & & & \\
2 & & & & \\
Sum $^{*}$ & 99 & 100 & 100 & 100 \\
\hline
\end{tabular}

Region 42a (Chromosome 15, $409.92 \mathrm{~kb}$ )

African-American

50 individuals, 100 SNPs, 16 blocks

\begin{tabular}{c|cccc|} 
\# of blocks & ran10 & $\operatorname{ran} 20$ & $\operatorname{ran} 30$ & $\operatorname{ran} 40$ \\
19 & & 2 & 10 & 5 \\
18 & & 7 & 12 & 19 \\
17 & & 18 & $\mathbf{4 2}$ & $\mathbf{5 3}$ \\
16 & & 38 & 34 & 23 \\
15 & 1 & 25 & 2 & \\
14 & 14 & 10 & & \\
13 & 24 & & & \\
12 & 30 & & & \\
11 & 22 & & & \\
10 & 5 & & & \\
9 & & & & \\
8 & & & & \\
7 & & & & \\
6 & & & & \\
5 & & & & \\
Sum $^{*}$ & 96 & 100 & 100 & 100 \\
& & & &
\end{tabular}

Region 31a (Chromosome 9, $181.98 \mathrm{~kb})$

African-American

50 individuals, 23 SNPs, 3 blocks

\begin{tabular}{c|cccc|} 
\# of blocks & ran10 & $\operatorname{ran} 20$ & $\operatorname{ran} 30$ & $\operatorname{ran} 40$ \\
5 & & 1 & & \\
4 & 2 & 23 & 23 & 29 \\
3 & 29 & 63 & 74 & 69 \\
2 & 64 & 13 & 3 & 2 \\
1 & & & & \\
Sum $^{*}$ & 95 & 100 & 100 & 100 \\
\cline { 2 - 5 } & & &
\end{tabular}

*Sum does not always add up to 100 . See results part for detailed explanation.

\section{Japanese \& Chinese}

42 individuals, 45 SNPs, 6 blocks

\begin{tabular}{|cccc|}
\hline $\operatorname{ran} 10$ & $\operatorname{ran} 20$ & $\operatorname{ran} 30$ & $\operatorname{ran} 40$ \\
& & & \\
& & & \\
& & 6 & 12 \\
1 & 36 & $\mathbf{7 5}$ & $\mathbf{8 8}$ \\
4 & $\mathbf{5 1}$ & 19 & \\
$\mathbf{5 1}$ & 13 & & \\
42 & & & \\
98 & 100 & 100 & 100 \\
\hline
\end{tabular}

Japanese \& Chinese

42 individuals, 99 SNPs, 14 blocks

\begin{tabular}{|cccc|}
\hline $\operatorname{ran} 10$ & ran20 & ran30 & $\operatorname{ran} 40$ \\
& & & \\
& & & \\
& & & \\
& & & \\
& & & 23 \\
& 5 & 13 & 49 \\
& 16 & 36 & 27 \\
13 & 33 & 37 & 1 \\
26 & 41 & 14 & \\
38 & 5 & & \\
14 & & & \\
1 & & & \\
92 & 100 & 100 & 100 \\
\hline
\end{tabular}

Japanese \& Chinese

42 individuals, 25 SNPs, 3 blocks

\begin{tabular}{|cccc|}
\hline $\operatorname{ran} 10$ & $\operatorname{ran} 20$ & $\operatorname{ran} 30$ & $\operatorname{ran} 40$ \\
& 3 & 1 & \\
& 9 & 12 & 4 \\
15 & $\mathbf{6 1}$ & $\mathbf{7 9}$ & $\mathbf{9 6}$ \\
$\mathbf{6 0}$ & 23 & 8 & \\
& & & \\
75 & 96 & 100 & 100 \\
\hline
\end{tabular}


we had few individuals or few markers included in the sample. Among the 99 simulated samples with HapBlock results, region 52a was partitioned into five blocks 17 times, six blocks 55 times, seven blocks 21 times, and eight blocks six times. If we focus on the trend of modes for each sample size based on 100 simulated samples, it is apparent that the number of blocks generally increases as we include more individuals in the sample. With the original 50 African-Americans, region 52a was partitioned into nine blocks. When we included only ten people, most of the times we obtained six blocks for this region. When we increased the sample size to 20 people, most of the times the region was partitioned into eight blocks.

When the sample size grew to 30 and 40, most of the times the region was partitioned into nine blocks, the same as that in the original dataset. Therefore, a minimum of 30 individuals is needed for this given set of markers to infer the number of blocks.

We also examined the sample size effect on the total number of Tag SNPs associated with block partitioning, and the results are summarised in Table 2. Similar to the results summarised in Table 1, the total number of Tag SNPs increases as the sample size increases. A shorter region with fewer SNPs, such as region 31a, seems to require fewer individuals than a longer region with more SNPs, such as regions $52 \mathrm{a}$ and $42 \mathrm{a}$, to identify a similar number of Tag SNPs as the original sample. In fact, the inferred number of blocks and Tag SNPs did not level off in region $42 \mathrm{a}$ in either population, indicating that our sample size may not have been adequate to define a set of Tag SNPs for this region. Statistical comparisons based

Table 2. Frequency of the total number of Tag SNPs when the number of individuals is varied in simulations

Region 52a (Chromosome 22, $237.22 \mathrm{~kb}$ )

African-American 50 individuals, 46 SNPs, total 19 Tag SNPs

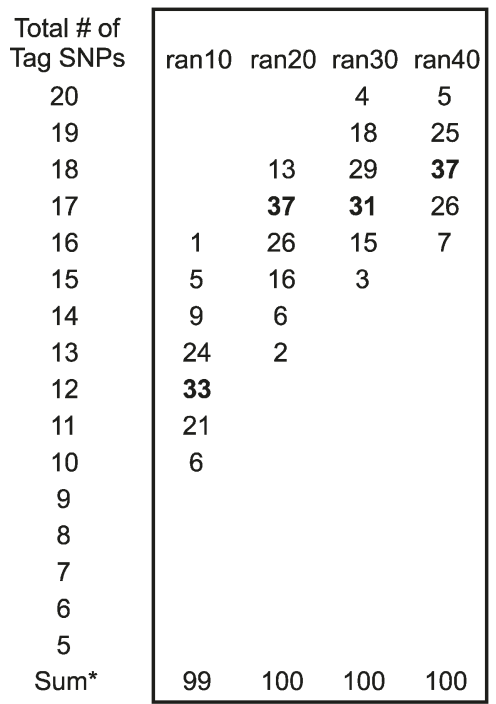

Region 31a (Chromosome 9, $181.98 \mathrm{~kb}$ )

African-American 50 individuals, 23 SNPs, total 6 Tag SNPs

\begin{tabular}{c|cccc|} 
Total \# of & \multicolumn{4}{c}{ Tag SNPs } \\
\cline { 2 - 4 } Tan10 & ran20 & ran30 & ran40 \\
8 & & 1 & 1 & \\
7 & 2 & 9 & 8 & 11 \\
6 & 5 & 32 & $\mathbf{4 8}$ & $\mathbf{5 2}$ \\
5 & 24 & $\mathbf{4 0}$ & 40 & 35 \\
4 & $\mathbf{5 1}$ & 16 & 3 & 2 \\
3 & 13 & 2 & & \\
Sum $^{*}$ & 95 & 100 & 100 & 100 \\
\hline
\end{tabular}

Japanese \& Chinese 42 individuals, 45 SNPs, total 10 Tag SNPs

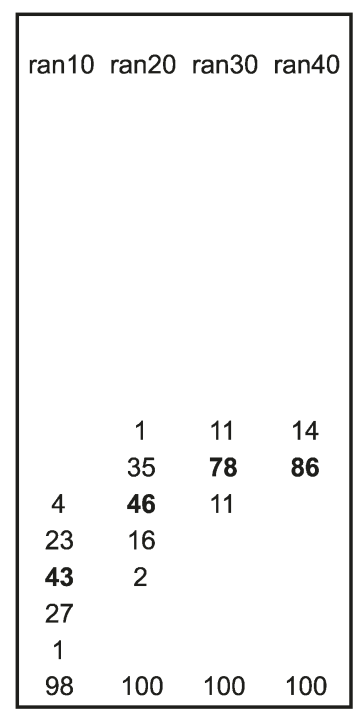

Japanese \& Chinese 42 individuals, 25 SNPs, total 6 Tag SNPs

\begin{tabular}{|cccc|}
\hline $\operatorname{ran} 10$ & $\operatorname{ran} 20$ & $\operatorname{ran} 30$ & $\operatorname{ran} 40$ \\
& 2 & & \\
& 5 & 8 & 5 \\
2 & 32 & 65 & 95 \\
6 & 15 & 10 & \\
28 & 26 & 13 & \\
39 & 16 & 4 & \\
75 & 96 & 100 & 100 \\
\hline
\end{tabular}

*Sum does not always add up to 100 . See results part for detailed explanation.

Region 42a (Chromosome 15, 409.92 kb)

African-American 50 individuals, 100 SNPs, total 33 Tag SNPS

Japanese \& Chinese 42 individuals, 99 SNPs, total 22 Tag SNPs

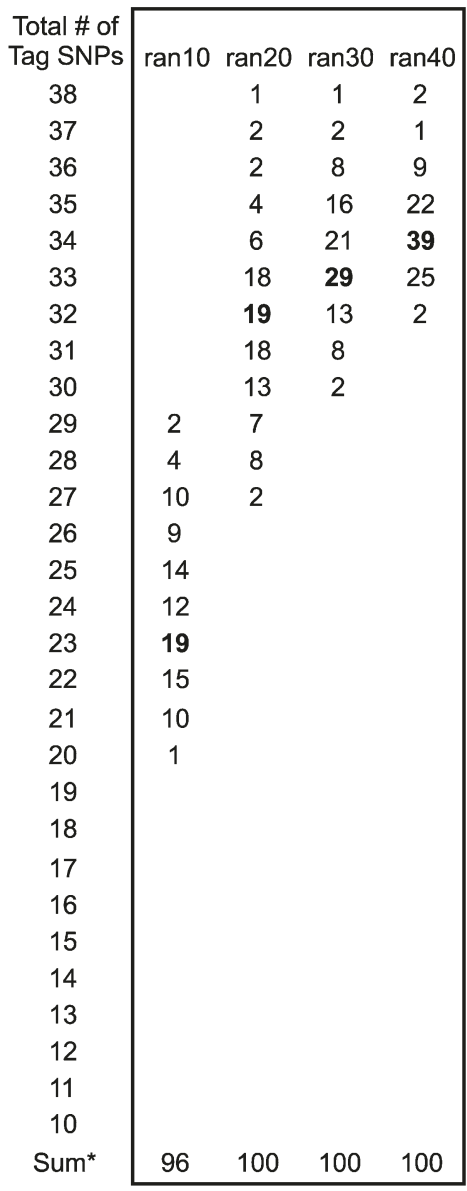




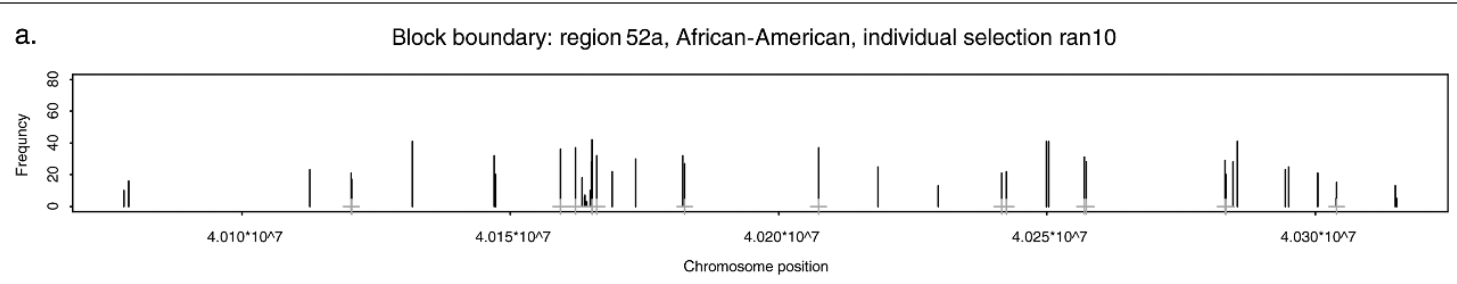

Block boundary: region 52a, African-American, individual selection ran20

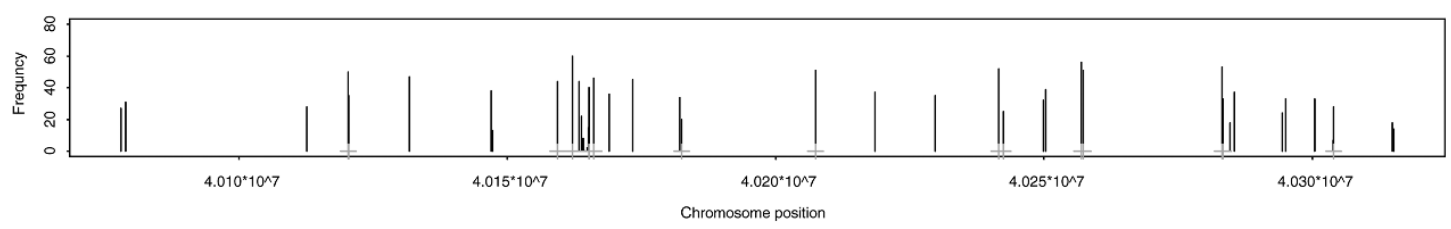

Block boundary: region 52a, African-American, individual selection ran30

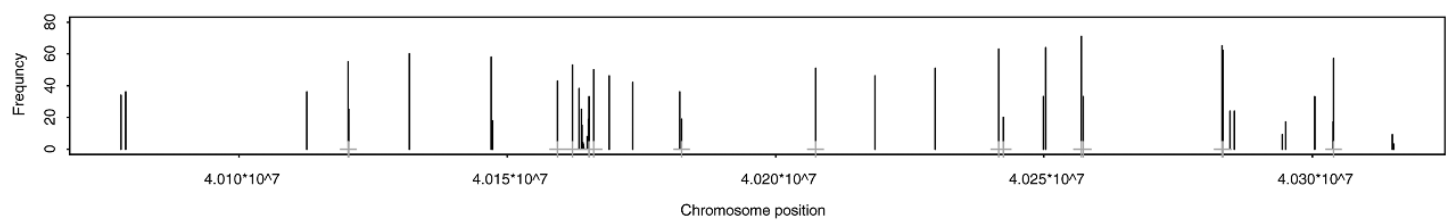

Block boundary: region52a, African-American, individual selection ran40

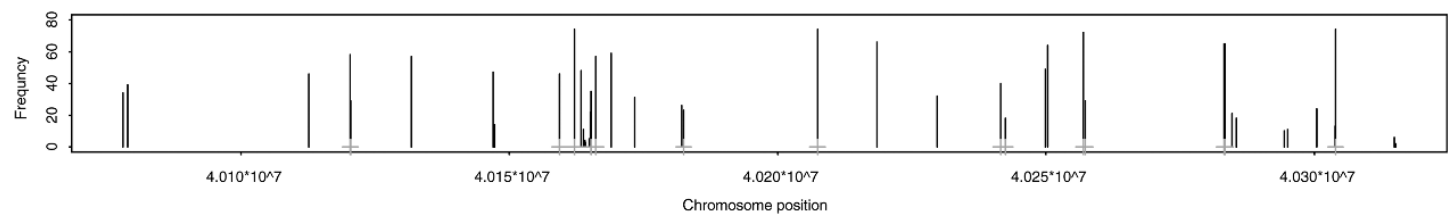

b. Block boundary: region52a Japanese \& Chinese, individual selection ran10

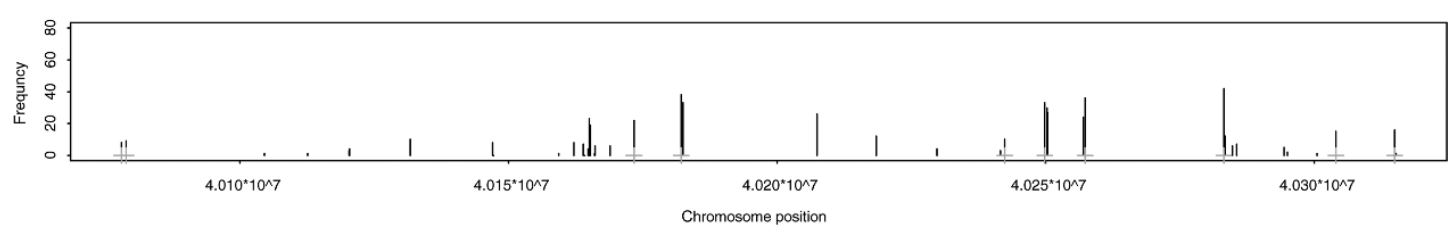

Block boundary: region 52a Japanese \& Chinese, individual selection ran20

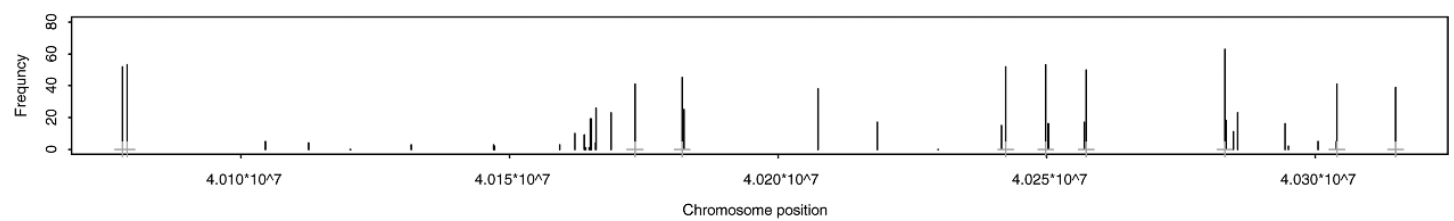

Block boundary: region 52a Japanese \& Chinese, individual selection ran30

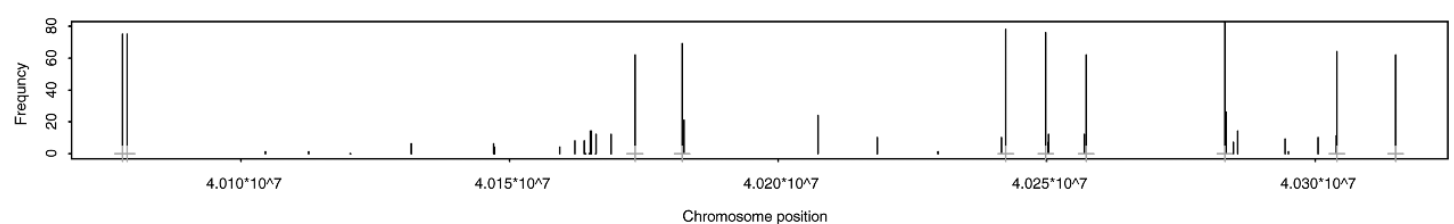

Block boundary: region 52a Japanese \& Chinese, individual selection ran40

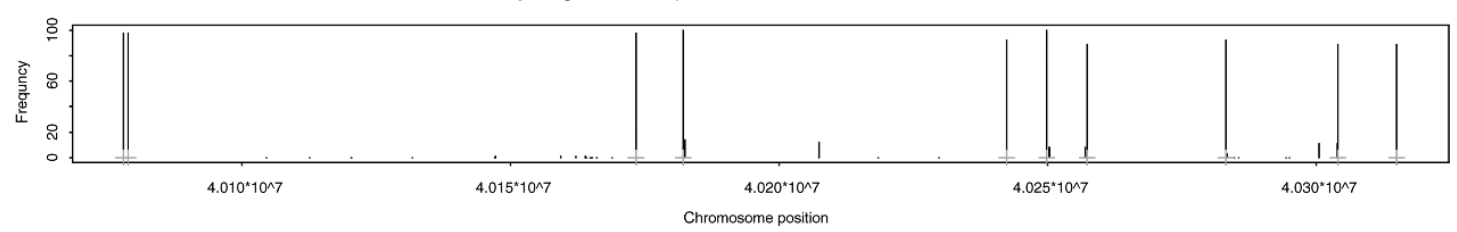

Figure I. Frequency of each SNP being selected as block boundary against its chromosomal location in individual selection for Region 52a. (a) African-American. (b) Japanese \& Chinese. + indicates the position of block boundaries using the original sample. 
on t-tests or Wilcoxon tests also indicated that there was a significant difference between the inferred block structures from samples of size 30 and those from samples of size 40 in region $52 \mathrm{a}$.

Using region 52a as an example, Figure 1 summarises the frequency of each SNP being used as block boundary against its chromosomal location across 100 simulated samples with 10, 20, 30 and 40 individuals, respectively. Although block boundaries differed from one sample to another (for samples consisting of the same number of individuals), when we pooled the results of 100 random selections, the overall patterns were very similar for samples of different sizes. The block boundaries in region $52 \mathrm{a}$ from the Japanese and Chinese samples were more clear-cut than those from the AfricanAmerican samples. The high frequency bars matched block boundary positions from those identified in the original 42 Japanese and Chinese people perfectly.

Detailed Tag SNP comparisons are more difficult than block boundary comparisons mainly because Tag SNPs are not unique. Usually there is more than one set of Tag SNPs in a block (see Appendix A for tag SNP example). In order to examine the impact of sample size on Tag SNP selections, we calculated the weighted frequency of each SNP being selected as a Tag SNP and plotted it against the SNPs in the combined order (see Appendix B for SNPs in the combined order due to differences between SNP sets between the two populations). Figure 2 summarises the results for Tag SNP selections for different sample sizes $(10,20,30$ and 40) and it can clearly be seen that similar sets of Tag SNPs were identified on average across all simulations for different sizes. Comparing these to the Tag SNPs from the original sample of 50 African-

Americans, we found that they were almost identical, with the exception of SNP numbers 20 and 45. Both of these had a relatively high frequency of being selected as Tag SNPs using randomly selected samples, but they did not show up in the Tag SNP list using the original sample. In addition, we found that most of the Tag SNPs selected for the Japanese and Chinese population also appeared on the Tag SNP list for the African-American population, but not vice versa, indicating that Tag SNPs for the Japanese and Chinese population

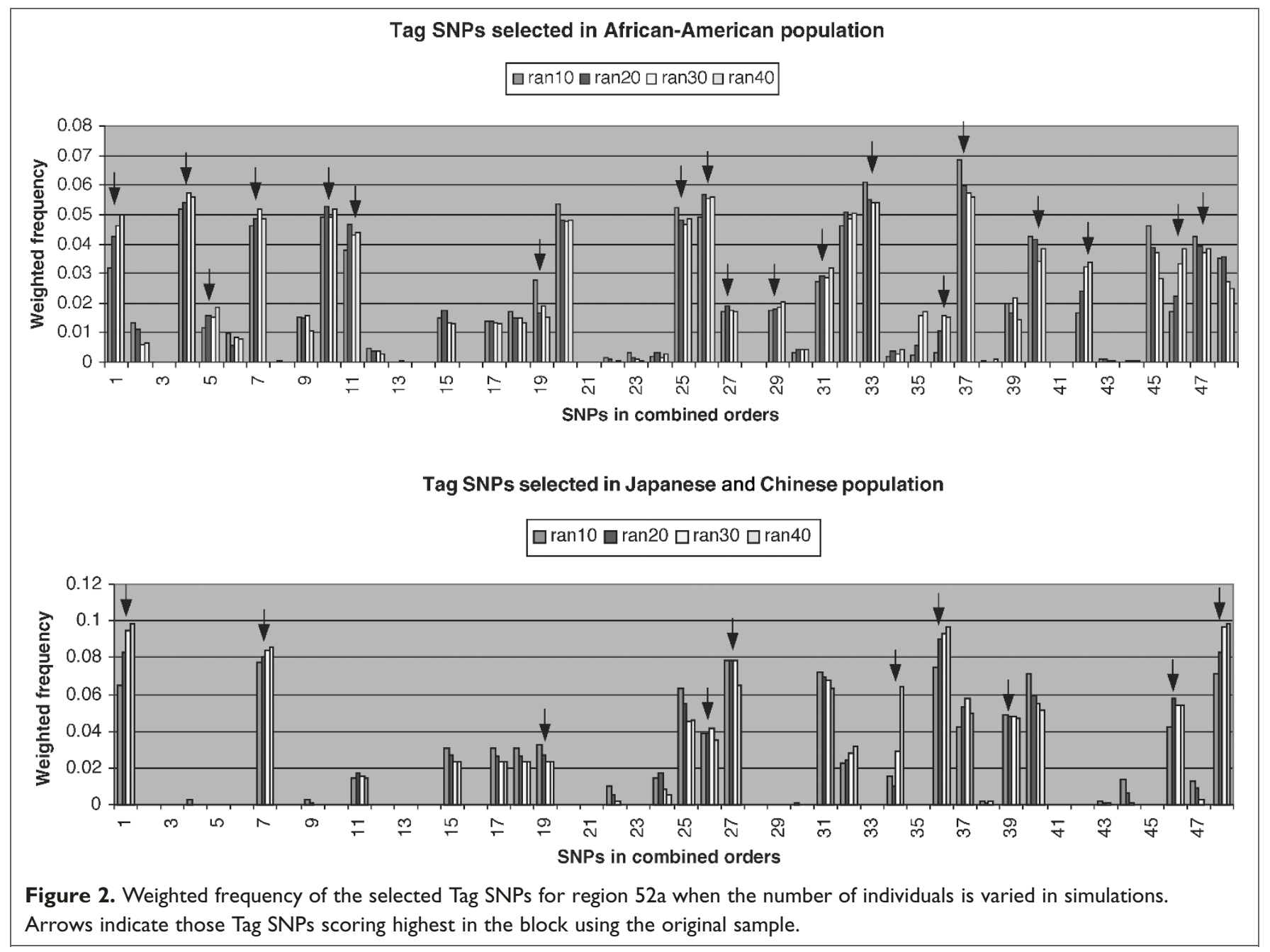


Table 3. Frequency of the number of blocks when the number of markers is varied in simulations

Region 52a (Chromosome 22, $237.22 \mathrm{~kb}$ )

African-American Region

50 individuals, 46 SNPs, 9 blocks

\begin{tabular}{c|cccc|} 
\# blocks & ran10 & ran20 & ran30 & ran40 \\
9 & & & & 11 \\
8 & & & & $\mathbf{7 4}$ \\
7 & & & 42 & 15 \\
6 & & 4 & 49 & \\
5 & & 40 & 9 & \\
4 & 3 & 54 & & \\
3 & 51 & 2 & & \\
2 & 45 & & & \\
Sum $^{*}$ & 99 & 100 & 100 & 100 \\
\hline
\end{tabular}

Region 42a (Chromosome 15, $409.92 \mathrm{~kb}$ )

African-American

50 individuals, 100 SNPs, 16 blocks

\begin{tabular}{c|cccc|} 
\# blocks & ran20 & ran40 & ran60 & ran80 \\
16 & & & & 14 \\
15 & & & 1 & 42 \\
14 & & & 7 & 37 \\
13 & & & 17 & 7 \\
12 & & & 48 & \\
11 & & 1 & 25 & \\
10 & & 13 & 2 & \\
9 & & 40 & & \\
8 & & 43 & & \\
7 & 1 & 3 & & \\
6 & 18 & & & \\
5 & 54 & & & \\
4 & 27 & & & \\
3 & & & & \\
2 & & & & \\
Sum $^{*}$ & 100 & 100 & 100 & \\
& & & &
\end{tabular}

Region 31a (Chromosome 9, 181.98 kb)

\section{African-American}

50 individuals, 23 SNPs, 3 blocks

\begin{tabular}{c|ccc|} 
\# blocks & ran10 & ran15 & ran20 \\
4 & & & 1 \\
3 & 2 & 24 & 84 \\
2 & 72 & 76 & 15 \\
1 & & & \\
Sum* & 74 & 100 & 100 \\
\hline
\end{tabular}

Japanese \& Chinese

42 individuals, 42 SNPs, 6 blocks

\begin{tabular}{|cccc|}
\hline $\operatorname{ran} 10$ & $\operatorname{ran} 20$ & $\operatorname{ran} 30$ & $\operatorname{ran40}$ \\
& & & \\
& & & 4 \\
& & 17 & 73 \\
& 9 & 56 & 22 \\
1 & 25 & 25 & 1 \\
22 & 51 & 2 & \\
63 & 15 & & \\
86 & 100 & 100 & 100 \\
\hline
\end{tabular}

Japanese \& Chinese

42 individuals, 99 SNPs, 14 blocks

\begin{tabular}{|cccc|}
\hline $\operatorname{ran20}$ & ran40 & ran60 & ran80 \\
& & & 1 \\
& & & 3 \\
& & & 16 \\
& & 2 & 31 \\
& & 8 & 31 \\
& 1 & 22 & 15 \\
& 3 & 38 & 3 \\
& 19 & 27 & \\
1 & 31 & 3 & \\
22 & 36 & & \\
$\mathbf{5 5}$ & 10 & & \\
21 & & & \\
1 & & & \\
100 & 100 & 100 & 100 \\
\hline
\end{tabular}

Japanese \& Chinese

42 individuals, 25 SNPs, 3 blocks

\begin{tabular}{|ccc|}
\hline $\operatorname{ran} 10$ & $\operatorname{ran} 15$ & $\operatorname{ran} 20$ \\
2 & 6 & 4 \\
31 & $\mathbf{5 8}$ & $\mathbf{9 2}$ \\
$\mathbf{4 8}$ & 36 & 4 \\
& & \\
81 & 100 & 100 \\
\hline
\end{tabular}

* Sum does not always add up to 100 . See results part for detailed explanation.

is largely a subset of those for the African-American population.

\section{Impact of marker selection}

Table 3 summarises the results of the number of blocks after we randomly selected: 10, 20, 30 and 40 SNPs for region 52a; 20, 40, 60 and $80 \mathrm{SNPs}$ for region 42a; and 10, 15 and 20 SNPs for region 31a. Simulated samples consisting of a random selection of five SNPs for region 31a crashed the HapBlock program every time, and therefore no results from this part of the study are shown in Table 3. It is apparent from this Table that as we included more SNP 
Table 4. Frequency of the total number of Tag SNPs when the number of markers is varied in simulations

Region 52a (Chromosome 22, $237.22 \mathrm{~kb}$ )

African-American 50 individuals, 46 SNPs, total 19 Tag SNPs

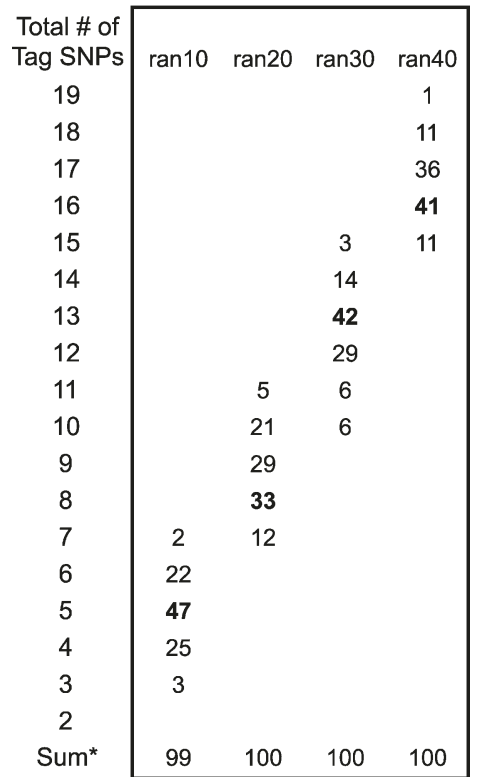

Japanese \& Chinese 42 individuals, 45 SNPs, total 10 Tag SNPs

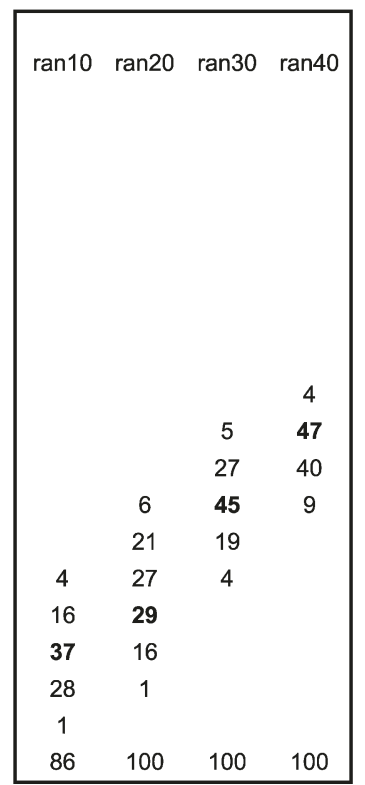

Region 31 a (Chromosome 9, $181.98 \mathrm{~kb})$

African-American

50 individuals, 23 SNPs, total 6 Tag SNPs

\begin{tabular}{c|ccc|} 
Total \# of & & & \\
Tag SNPs & ran10 & ran15 & ran20 \\
6 & & & 27 \\
5 & 2 & 18 & 57 \\
4 & 28 & 66 & 16 \\
3 & 44 & 16 & \\
2 & & & \\
Sum $^{*}$ & 74 & 100 & 100 \\
\hline
\end{tabular}

* Sum does not always add up to 100 . See results part for detailed explanation.

markers in our sample, the number of blocks continued to grow, and there was evidence that the inferred haplotype structures would have continued to change if more markers had been included.

As for the number of Tag SNPs, Table 4 clearly shows that, as we included more SNP markers in our sample, the total number of Tag SNPs also continued to grow, and did not show any sign of stabilisation.

To answer the question of why denser marker sets usually give rise to more, smaller blocks than is the case for sparser marker sets, we studied chromosomal region 52a in the African-American population in detail. Figure 3 shows two representative patterns of how region 52a was partitioned into

Region 42a (Chromosome 15, 409.92 kb)

African-American

50 individuals, 100 SNPs, total 33 Tag SNPs

\begin{tabular}{|c|c|c|c|c|}
\hline $\begin{array}{c}\text { Total \# of } \\
\text { Tag SNPs }\end{array}$ & $\operatorname{ran} 20$ & $\operatorname{ran} 40$ & $\operatorname{ran} 60$ & $\operatorname{ran} 80$ \\
\hline 32 & & & & 3 \\
\hline 31 & & & & 9 \\
\hline 30 & & & & 18 \\
\hline 29 & & & & 28 \\
\hline 28 & & & & 27 \\
\hline 27 & & & 2 & 10 \\
\hline 26 & & & 11 & 3 \\
\hline 25 & & & 15 & 1 \\
\hline 24 & & & 23 & 1 \\
\hline 23 & & 1 & 30 & \\
\hline 22 & & 1 & 13 & \\
\hline 21 & & 1 & 4 & \\
\hline 20 & & 6 & 2 & \\
\hline 19 & & 8 & & \\
\hline 18 & & 20 & & \\
\hline 17 & & 26 & & \\
\hline 16 & & 20 & & \\
\hline 15 & & 14 & & \\
\hline 14 & & 3 & & \\
\hline 13 & & & & \\
\hline 12 & 6 & & & \\
\hline 11 & 18 & & & \\
\hline 10 & 31 & & & \\
\hline 9 & 30 & & & \\
\hline 8 & 15 & & & \\
\hline 7 & & & & \\
\hline 6 & & & & \\
\hline 5 & & & & \\
\hline 4 & & & & \\
\hline Sum* & 100 & 100 & 100 & 100 \\
\hline
\end{tabular}

Japanese \& Chinese 42 individuals, 99 SNPs, total 22 Tag SNPs

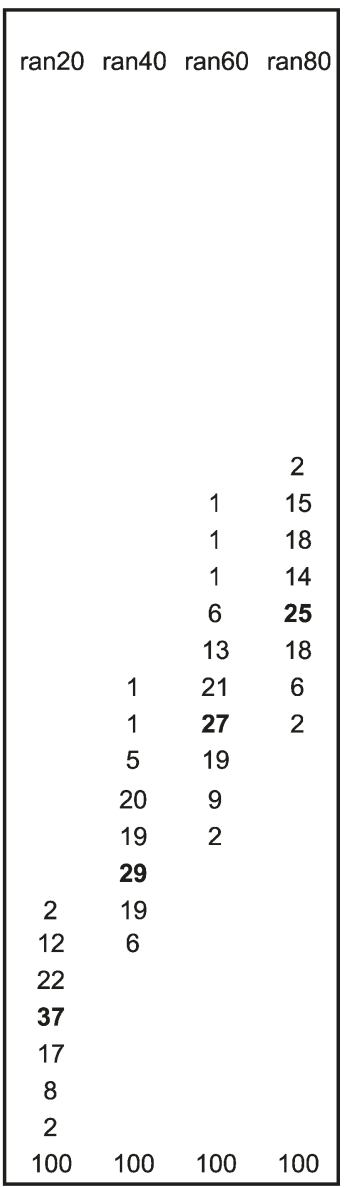

blocks using 10, 20, 30 and 40 sequentially-selected SNP markers, as well as the original 46 SNP marker set. Both marker sets of size 10 generated three blocks, with one set consisting of SNPs number 2, 8, 19, 21, 24, 30, 36, 42, 43 and 46, and the other set consisting of SNPs number 5, 6, 11, 15, 22, 23, 24, 25, 40 and 45. The blank space between the blocks is due to the lack of information regarding which block the SNPs belong to. By adding ten more SNPs to both marker sets, the two 20-marker sets generated five blocks, as shown in Figures $3 \mathrm{a}$ and $3 \mathrm{~b}$. As we included additional SNPs in the marker set within this region, i.e. as we increased the marker density, the number of blocks increased for two reasons. First, the old large blocks at lower densities are often broken into 
a.

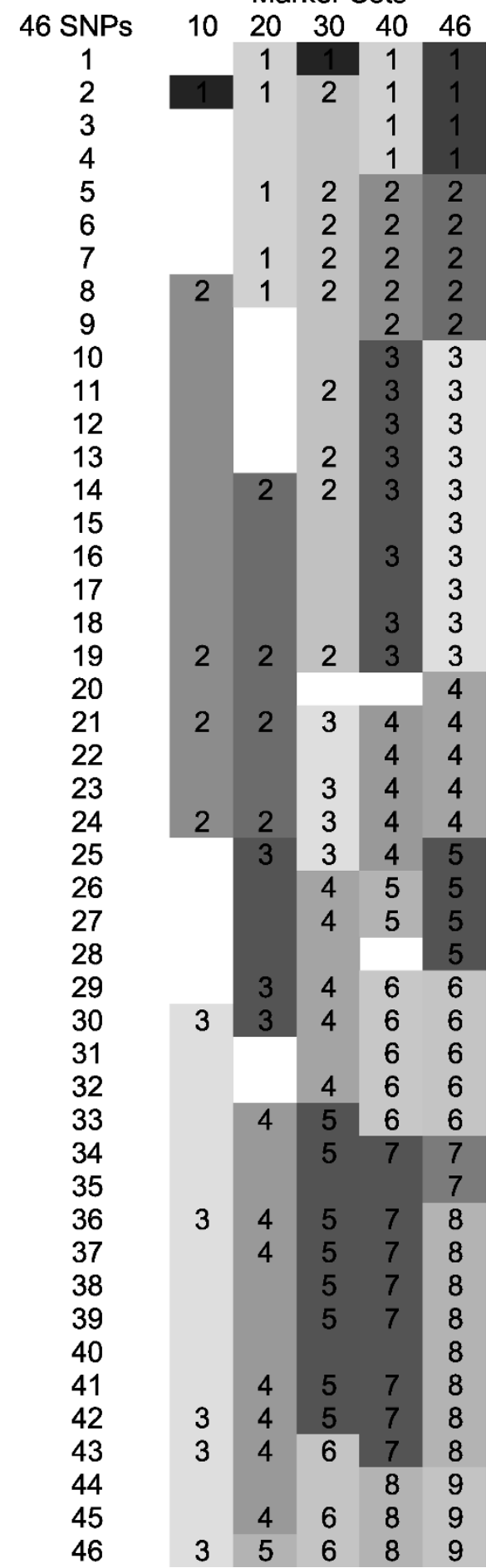

b.

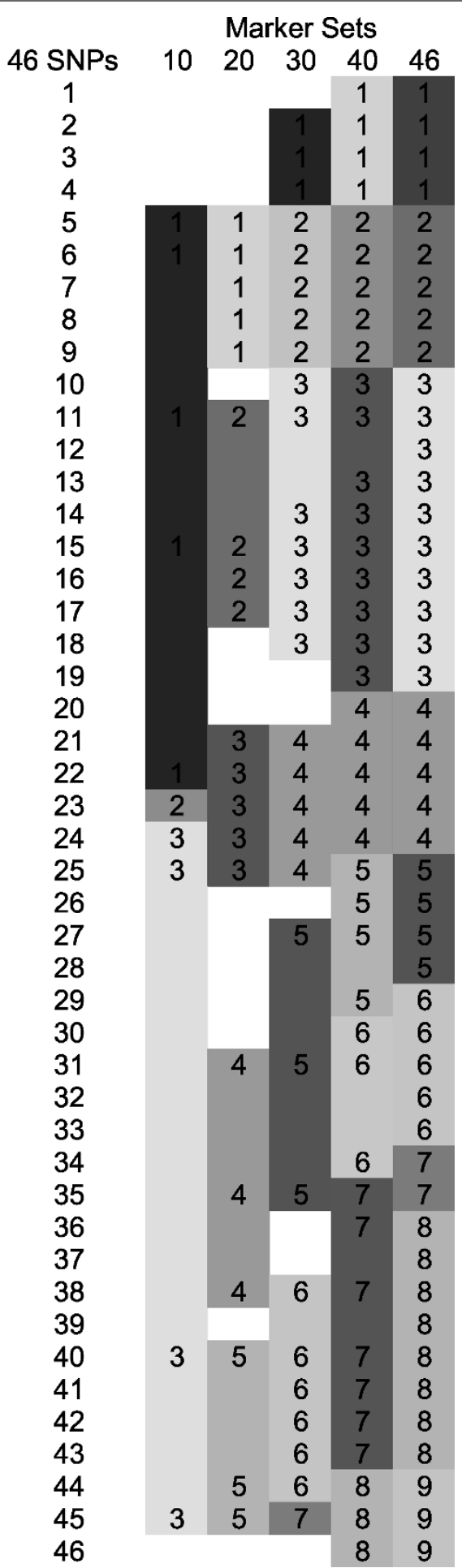

Figure 3. Two representative samples of block partitions on region 52a using 46 original SNP markers from the African-American population and 10,20,30, 40 marker sets generated by partially fixed marker selection method. Each block is denoted by the shaded areas above. Labels such as ' 1 ', ' 2 ', etc on each shaded area indicate the position where a particular SNP was selected in the marker set, as well as which block it is on.

smaller pieces at higher density. For example, in Figure 3a, block 7 in marker set 40 became block 7 and block 8 when two more SNPs (numbers 35 and 40) were added to this region. Secondly, new blocks emerged from areas where there was a lack of information due to the lack of markers in the smaller marker set, such as block 3 in marker set 20 in 
Figure $3 \mathrm{a}$ and block 1 in marker set 30 in Figure $3 \mathrm{~b}$. The block boundaries were obviously not random but were in fact quite consistent across different marker sets.

\section{Discussion}

Our studies have clearly demonstrated that sample size and marker selection have a significant impact on the number of blocks and the total number of Tag SNPs inferred from a population sample. As we include more individuals in our sample, both the number of blocks and the total number of Tag SNPs increase. For a shorter region with fewer SNP markers, like region 31a (181.98 kb, 23 SNPs), 20 people may be adequate to infer the haplotype patterns, while for a longer region with more SNP markers, such as $52 \mathrm{a}(237.22 \mathrm{~kb}, 46$ SNPs) and 42a (409.92 kb, 100 SNPs), the required sample size may be 30 or more. The minimal sample size needed for a reliable haplotype structure inference clearly depends on the structure of the region being investigated. Although the patterns of block boundary and the set of Tag SNPs selected look very similar on average across all sample sizes, there is more variation from one simulated sample to another when the sample size is small. In addition, the set of Tag SNPs selected in the Japanese and Chinese population seems to be a subset of those in the African-American population. ${ }^{8}$ This observation, however, may be due to the ascertainment of the specific set of markers being examined in the original study.

Our marker selection results demonstrate that the number of blocks and the total number of Tag SNPs increase as more SNP markers are included. In addition, our results indicate that we would need to include more SNP markers in these regions in order to draw a valid conclusion on the number of blocks and Tag SNPs. The number of SNPs needed for a reliable inference on the haplotype structures may be a function of both the region and the specific population under study.

Another issue to bear in mind is that our haplotypes were inferred from genotype data, not directly observed. Although the accuracy is quite high, greater than 80 per cent, ${ }^{17}$ it is likely that the results may differ if different algorithms are used to reconstruct individual haplotypes. In addition, the inaccuracy in haplotype inference may contribute to the observed sample size effect. It should also be noted that the specific set of parameters used in the HapBlock program in our analysis to infer blocks and Tag SNPs does not affect the general patterns for the impact of the sample size and marker selection on the inferred haplotype structures (results not shown).

In summary, our study indicates that sample size and marker selection have a significant impact on the inferred haplotype structures reflected in the haplotype blocks and Tag SNPs. Although haplotype blocks may be an over-simplistic representation of the haplotype structures, ${ }^{14}$ we hypothesise that the impact would have been equally significant if we had used other approaches to analysing haplotype structures in the human genome. In order to draw valid conclusions on haplotype block structure, we need a relatively large sample size and a dense marker panel and we need to make adaptive adjustments according to the specific region and specific population to be studied.

\section{Acknowledgments}

We thank Dr Kui Zhang for his generous support on the HapBlock program, two reviewers for their constructive comments, and Gabriel and colleagues for making their datasets available. This work was supported in part by NIH grant R01 GM59507 to H.Z. 


\section{Appendix A}

\section{Region 52a (Chromosome 22, $237.22 \mathrm{~kb}$ )}

\begin{tabular}{|c|c|c|c|c|c|}
\hline \\
\hline \multicolumn{6}{|c|}{ \# of blocks 9 total \# of Tag SNPs 19} \\
\hline BlockID & NumTagSNP & StartPos & EndPos & BlockSize & NumHap \\
\hline Block_000I & 3 & I & 4 & 4 & 100 \\
\hline Block_0002 & 2 & 5 & 9 & 5 & 100 \\
\hline Block_0003 & 2 & 10 & 19 & 10 & 100 \\
\hline Block_0004 & 2 & 20 & 24 & 5 & 100 \\
\hline Block_0005 & 2 & 25 & 28 & 4 & 100 \\
\hline Block_0006 & 2 & 29 & 33 & 5 & 100 \\
\hline Block_0007 & 2 & 34 & 35 & 2 & 100 \\
\hline Block_0008 & 2 & 36 & 43 & 8 & 100 \\
\hline Block_0009 & 2 & 44 & 46 & 3 & 100 \\
\hline
\end{tabular}

Tag SNP for block_000I

$\begin{array}{llll}\mathrm{I} & 4 & 5 & 0.95825 \\ \mathrm{I} & 4 & 5 & 0.95825-\mathrm{I}\end{array}$

Tag SNP for block_0002

$\begin{array}{lll}7 & 10 & 0.94339 \\ 9 & 10 & 0.90594 \\ 7 & 10 & 0.94339-I^{*}\end{array}$

Tag SNP for block_0003

II $\quad$ I5 $\quad 0.93446$

$\begin{array}{lll}\text { II } & 17 & 0.93107\end{array}$

II $\quad 18 \quad 0.93234$

II $\quad 19 \quad 0.9346$

$15 \quad 20 \quad 0.92013$

$17 \quad 20 \quad 0.91561$

$18 \quad 20 \quad 0.91455$

$19 \quad 20 \quad 0.92754$

II $\quad$ I9 $0.9346-$ I*

Tag SNP for block_0004

$25 \quad 26 \quad 0.90927$

$25 \quad 26 \quad 0.90927-I^{*}$

$\dagger$ Tag SNPs are in combined order.

* - I lines indicate the Tag SNPs that scored the highest in each block by the HapBlock program.
Tag SNP for block_0005

$\begin{array}{lll}27 & 29 & 0.91095 \\ 27 & 29 & 0.91095-I^{*}\end{array}$

Tag SNP for block_0006

$\begin{array}{lll}31 & 33 & 0.90816 \\ 32 & 33 & 0.90614 \\ 31 & 33 & 0.90816-I^{*}\end{array}$

Tag SNP for block_0007

$\begin{array}{lll}36 & 37 & 1 \\ 36 & 37 & 1-I^{*}\end{array}$

Tag SNP for block_0008

$\begin{array}{lll}40 & 42 & 0.90181 \\ 40 & 42 & 0.90181-I^{*}\end{array}$

Tag SNP for block_0009

$\begin{array}{lll}46 & 47 & 0.93096 \\ 46 & 48 & 0.92839 \\ 46 & 47 & 0.93096-I^{*}\end{array}$


Population C (Japanese \& Chinese) ${ }^{\dagger}$

\# of blocks $=6 \quad$ Total \# of TagSNPs $=10$

$\begin{array}{llcccc}\text { BlockID } & \text { NumTagSNP } & \text { StartPos } & \text { EndPos } & \text { BlockSize } & \text { NumHap } \\ \text { Block_000I } & \mathrm{I} & \mathrm{I} & \mathrm{I} & \mathrm{I} & 84 \\ \text { Block_0002 } & 2 & 2 & 22 & 21 & 84 \\ \text { Block_0003 } & 2 & 23 & 29 & 7 & 84 \\ \text { Block_0004 } & 2 & 30 & 34 & 5 & 84 \\ \text { Block_0005 } & 2 & 35 & 43 & 9 & 84 \\ \text { Block_0006 } & \mathrm{I} & 44 & 45 & 2 & 84\end{array}$

Tag SNP for block_000I

I $\quad 1.00000$

I $\quad 1.00000-I^{*}$

Tag SNP for block_0004

$32 \quad 36 \quad 0.90335$

$34 \quad 36 \quad 0.9073$

$34 \quad 36 \quad 0.9073-\left.\right|^{*}$

Tag SNP for block_0002

$\begin{array}{lll}7 & 15 & 0.96085\end{array}$

$\begin{array}{lll}7 & 17 & 0.96085\end{array}$

$\begin{array}{lll}7 & 18 & 0.96085\end{array}$

$7 \quad 19 \quad 0.96085$

$7 \quad 15 \quad 0.96085-1 *$

Tag SNP for block_0005

$\begin{array}{lll}37 & 39 & 0.93032\end{array}$

$\begin{array}{lll}37 & 40 & 0.92593\end{array}$

$39 \quad 46 \quad 0.93265$

$40 \quad 46 \quad 0.92716$

Tag SNP for block_0003

$\begin{array}{lll}25 & 27 & 0.9219\end{array}$

$39 \quad 46 \quad 0.93265-$ I*

$25 \quad 31 \quad 0.90516$

$\begin{array}{lll}26 & 27 & 0.92676\end{array}$

$26 \quad 31 \quad 0.91236$

$27 \quad 31 \quad 0.92645$

$26 \quad 27 \quad 0.92676-I^{*}$

† Tag SNPs are in combined order.

$*_{-}$I lines indicate the Tag SNPs that scored the highest in each block by the HapBlock program. 


\section{Appendix B}

\begin{tabular}{|c|c|c|c|c|c|c|}
\hline SNP_ID & $\begin{array}{l}\text { COMBINED } \\
\text { ORDER }\end{array}$ & $\begin{array}{l}\text { POP_B } \\
\text { ORDER }\end{array}$ & $\begin{array}{l}\text { POP_C } \\
\text { ORDER }\end{array}$ & CHROM_POS & POP_B BLOCK & POP_C BLOCK \\
\hline 110924 & I & 1 & 1 & 40077996 & Block_000I & Block_000I \\
\hline 110926 & 2 & 2 & 2 & 40078865 & Block_000I & Block_0002 \\
\hline 110525 & 3 & NA & 3 & 40104585 & NA & Block_0002 \\
\hline I I 0527 & 4 & 3 & 4 & $40 I 12652$ & Block_000I & Block_0002 \\
\hline II 0528 & 5 & 4 & 5 & 40120338 & Block_000I & Block_0002 \\
\hline 110529 & 6 & 5 & 6 & 40120419 & Block_0002 & Block_0002 \\
\hline 3884 & 7 & 6 & 7 & 40131747 & Block_0002 & Block_0002 \\
\hline II 7587 & 8 & 7 & 8 & $40|4703|$ & Block_0002 & Block_0002 \\
\hline 117590 & 9 & 8 & 9 & 40147256 & Block_0002 & Block_0002 \\
\hline 91037 & 10 & 9 & 10 & 40159355 & Block_0002 & Block_0002 \\
\hline 82256 & II & 10 & 11 & 40162170 & Block_0003 & Block_0002 \\
\hline 117575 & 12 & 11 & NA & 40163399 & Block_0003 & NA \\
\hline II 7578 & 13 & 12 & NA & 40163843 & Block_0003 & NA \\
\hline 3943 & 14 & 13 & 12 & 40163920 & Block_0003 & Block_0002 \\
\hline 2442 & 15 & 14 & 13 & 40164108 & Block_0003 & Block_0002 \\
\hline 117580 & 16 & 15 & 14 & 40164192 & Block_0003 & Block_0002 \\
\hline | I758| & 17 & 16 & 15 & 40164236 & Block_0003 & Block_0002 \\
\hline II 7582 & 18 & 17 & 16 & 40164840 & Block_0003 & Block_0002 \\
\hline II 7583 & 19 & 18 & 17 & 40165138 & Block_0003 & Block_0002 \\
\hline 37728 & 20 & 19 & 18 & 40165262 & Block_0003 & Block_0002 \\
\hline 14523 & 21 & NA & 19 & 40166038 & NA & Block_0002 \\
\hline 82025 & 22 & 20 & 20 & 40166144 & Block_0004 & Block_0002 \\
\hline 84395 & 23 & 21 & 21 & $4016897 \mid$ & Block_0004 & Block_0002 \\
\hline 117586 & 24 & 22 & 22 & 40173352 & Block_0004 & Block_0002 \\
\hline 117592 & 25 & 23 & 23 & $40|82| 4 \mid$ & Block_0004 & Block_0003 \\
\hline 117593 & 26 & 24 & 24 & 40182498 & Block_0004 & Block_0003 \\
\hline 117596 & 27 & 25 & 25 & 40207457 & Block_0005 & Block_0003 \\
\hline 26726 & 28 & 26 & 26 & 40218483 & Block_0005 & Block_0003 \\
\hline 16893 & 29 & 27 & 27 & 40229786 & Block_0005 & Block_0003 \\
\hline 11692 & 30 & 28 & 28 & $4024 I 57 \mid$ & Block_0005 & Block_0003 \\
\hline 117608 & 31 & 29 & 29 & 40242422 & Block_0006 & Block_0003 \\
\hline 32936 & 32 & 30 & 30 & 40249849 & Block_0006 & Block_0004 \\
\hline 117566 & 33 & 31 & 31 & 40250303 & Block_0006 & Block_0004 \\
\hline 44133 & 34 & 32 & 32 & 40250387 & Block_0006 & Block_0004 \\
\hline II 7567 & 35 & 33 & 33 & 40256951 & Block_0006 & Block_0004 \\
\hline 23139 & 36 & 34 & 34 & 40257384 & Block_0007 & Block_0004 \\
\hline | | $868 \mid$ & 37 & 35 & 35 & 40283200 & Block_0007 & Block_0005 \\
\hline 99869 & 38 & 36 & 36 & 40283420 & Block_0008 & Block_0005 \\
\hline 2584 & 39 & 37 & 37 & 40284703 & Block_0008 & Block_0005 \\
\hline 118669 & 40 & 38 & 38 & 40285521 & Block_0008 & Block_0005 \\
\hline II 8674 & 41 & 39 & 39 & 40294440 & Block_0008 & Block_0005 \\
\hline 30109 & 42 & 40 & 40 & 40295018 & Block_0008 & Block_0005 \\
\hline 118676 & 43 & 41 & $4 I$ & 40300494 & Block_0008 & Block_0005 \\
\hline 88347 & 44 & 42 & NA & 40303907 & Block_0008 & Block_0005 \\
\hline 118679 & 45 & 43 & 42 & 40303949 & Block_0008 & NA \\
\hline 88348 & 46 & 44 & 43 & 40303993 & Block_0009 & Block_0005 \\
\hline 3742 & 47 & 45 & 44 & 40314969 & Block_0009 & Block_0006 \\
\hline 54 & 48 & 46 & 45 & 40315218 & Block_0009 & Block_0006 \\
\hline
\end{tabular}




\section{References}

1. Kruglyak, L. and Nickerson, D.A. (2001), 'Variation is the spice of life', Nat. Genet. Vol. 27, pp. 234-236.

2. Sachidanandam, R., Eeissman, D., Schmidt, S.C. et al. (2001), 'A map of human genome sequence variation containing 1.42 million single nucleotide polymorphisms', Nature Vol. 409, pp. 928-933.

3. Venter, J.C., Adams, N.D., Myers, E.W. et al. (2001), 'The sequence of the human genome', Science Vol. 291, pp. 1304-1351.

4. Stephens, J.C., Schneider, J.A., Tanguay, D.A. et al. (2001), 'Haplotype variation and linkage disequilibrium in 313 human genes', Science Vol. 293 pp. $489-493$.

5. Daly, M.J., Rioux, J.D., Schaffnel, S.F. et al. (2001), 'High-resolution haplotype structure in the human genome', Nat. Genet. Vol. 29, pp. 229-232.

6. Patil, N., Berno, A.J., Hurds, D.A. et al. (2001), 'Blocks of limited haplotype diversity revealed by high-resolution scanning of human chromosome', Science Vol. 294, pp. 1719-1723.

7. Dawson, E., Abecasis, G.R., Bumpstead, S. et al. (2002), 'A first-generation linkage disequilibrium map of human chromosome 22', Nature Vol 418, pp. 544-548.

8. Gabriel, S.B., Scnaffnel, S.F., Nguyen, H. et al. (2002), 'The structure of haplotype blocks in the human genome', Science Vol. 296, pp. 2225-2229.

9. Jeffreys, A.J., Kauppi, L. and Neumann, R. (2001), 'Intensely punctate meiotic recombination in the class II region of the major histocompatibility complex', Nat. Genet. Vol. 29, pp. 217-222.
10. Zhang, K., Calabrese, P., Nordborg, M. and Sun, F. (2002), 'Haplotype block structure and its applications to association studies: Power and study designs', Am. J. Hum. Genet. Vol. 71, pp. $1386-1394$

11. Reich, D.E., Cargill, M., Bolk, S. et al. (2001), 'Linkage disequilibrium in the human genome', Nature Vol. 411, pp. 199-204.

12. Zhang, K., Deng, H., Chen, T. et al. (2002), 'A dynamic programming algorithm for haplotype block partitioning', Proc. Natl. Acad. Sci. USA Vol. 99, pp. 7335-7339.

13. Wang, N., Akey, J.M., Zhang, K. et al. (2002), 'Distribution of recombination crossovers and the origin of haplotype blocks: The interplay of population history, recombination, and mutation', Am. J. Hum. Genet. Vol. 71, pp. 1227-1234.

14. Wall, J.D. and Pritchard, J.K. (2003), 'Haplotype blocks and linkage disequilibrium in the human genome', Nat. Rev. Genet. Vol. 4, pp. 587-597.

15. Wall, J.D. and Pritchard, J.K. (2003), 'Assessing the performance of the haplotype block model of linkage disequilibrium', Am. J. Hum. Genet. Vol. 73 , pp. $502-515$.

16. Johnson, G.C., Esposito, L., Barratt, B.J. et al. (2001), 'Haplotype tagging for the identification of common disease genes', Nat. Genet. Vol. 29, pp. $233-237$.

17. Stephens, M., Smith, N.J. and Donnelly, P. (2001), 'A new statistical method for haplotype reconstruction from population data', Am. J. Hum. Genet. Vol. 68, pp. 978-989. 